\title{
Optimal Inspection Strategy Planning for Geometric Tolerance Verification
}

\author{
Giovanni Moroni ${ }^{\mathrm{a}}$, Stefano Petrò ${ }^{\mathrm{a}, *}$ \\ ${ }^{a}$ Sezione di Tecnologie Meccaniche e Sistemi di Produzione, Dipartimento di Meccanica, \\ Politecnico di Milano, Via La Masa 1, 20156, Milano, Italy
}

\begin{abstract}
Two features characterize a good inspection system: it is accurate, and compared to the manufacturing cost, it is not expensive. Unfortunately, few measuring systems posses both these characteristics, i.e. low uncertainty comes with a cost. But also high uncertainty comes with a cost, because measuring systems with high uncertainty tend to generate more inspection errors, which come with a cost.

In the case of geometric inspection, the geometric deviation is evaluated from a cloud of points sampled on a part. Therefore, not only the measuring device has to be selected, but also the sampling strategy has to be planned, i.e. the sampling point cloud size and where points should be located on the feature to inspect have to be decided. When the measuring device is already available, as it often happens in geometric measurement, where most instruments are flexible, an unwise strategy planning can be the largest uncertainty contributor.

In this work, a model for the evaluation of the overall inspection cost is proposed. The optimization of the model can lead to an optimal inspection strategy in economic sense. However, the model itself is based on uncertainty evaluation, in order to assess the impact of measurement error on inspection cost. Therefore, two methodologies for evaluating the uncertainty will be proposed. These methodologies will be focused on the evaluation of the contribution of
\end{abstract}

\footnotetext{
${ }^{*}$ Correspondig Author. Tel +39 02 23998530. Fax +3902 23998585.

Email addresses: giovanni.moroni@polimi.it (Giovanni Moroni), stefano.petro@polimi.it (Stefano Petrò)
}

Preprint submitted to Elsevier

January 9, 2015 
the sampling strategy to the uncertainty. Finally, few case studies dealing with the inspection planning for a Coordinate Measuring Machine will be proposed. Keywords: Geometric Inspection; Geometric Deviation; Uncertainty; Sampling Strategy; Inspection Cost Model

\section{Introduction}

For any company, quality is one of the main factors in the competition to become the leader of the sector. Even if quality has often been considered only as "product quality", "process quality" should be considered, too, because a good process usually produces good products with even lower costs. Anyway, to ensure goodness of product/process quality, some control has to be performed, i.e. a quality system has to be established. Every quality system is essentially based on the observation of the product/process: a quality characteristic is measured, and from this measurement the behavior of the system is decided to be "good" or "not good". This may imply also that a defective part is rejected, or that the whole process has to be checked and adjusted, if required. From now on, just the problem of checking parts will be considered. The possibility of rejecting a part due to the quality inspection leads to some unexpected costs. Inspection of parts consists in comparing the actual result of some measurement performed on it with a "specification limit" $S L$. Due to measurement error [1] it is possible that a good part (namely a part complying with the $S L$ ) is rejected (type A error), or a defective part is accepted (type B error) [2]. Both these types of errors come with a cost.

Inspection error probability is strictly related to uncertainty [3]. But high accuracy measurements are more expensive than low accuracy measurements, so reducing inspection errors frequency (which implies the choice of low uncertainty measurements) will increase measurement costs: a trade-off between inspection error costs and measurement costs has to be defined. 


\subsection{Geometric Tolerances and Compliance to Tolerances}

In mechanical engineering, a quite common specification is a "geometric tolerance" $[4,5]$. A geometric tolerance states how much a real part can differ from its ideal, designed geometry; therefore, a geometric tolerance usually defines only an upper specification limit for the geometric deviation. Geometric tolerances importance is increasing because of the performances the market requires to mechanical systems: if dimensional tolerancing may suffice simple parts to ensure functionality of simple parts, more complex fits require well-structured definitions.

Of course, the check of a geometric tolerance involves the estimate of the geometric deviation, and an uncertainty evaluation has to be proposed for this estimate. Some international series of standards have been proposed giving guidelines for this evaluation, like ASME B89.7.3 [6, 7, 8] and ISO $14253[9,10$, 11]. In particular, ASME B89.7.3.1 [6] and ISO 14253-1 [9] propose "guidelines for decision rules" for "considering uncertainty in determining conformance to specifications". A decision rule should essentially identify three regions for the measurement result $[12,13]$ :

1. Conformance zone. If the measurement result falls in this zone, then the related part is accepted.

2. Non-conformance zone. If the measurement result falls in this zone, then the related part is rejected.

3. Uncertainty range. If the measurement result falls in this zone, it is not possible to state whether the part conforms or not to the tolerance.

ASME B89.7.3.1 and ISO 14253-1 differ in that the ASME standard gives only guidelines on how to choose these regions, because the selection of a decision rule is considered a business decision, and the flexibility of having a continuum of rules ranging from stringent to relaxed acceptance or rejection is needed in order to satisfy a broad range of industries; ISO standard instead rigidly states that a part should be accepted if its geometric deviation estimate is lower than the geometric tolerance reduced by the expanded uncertainty $U$, 
and rejected if its geometric deviation estimate is greater than the geometric tolerance augmented by $U$. The region $(L S-U, L S+U)$ is the uncertainty range (Fig. 1). In particular, the standard states that, if a supplier has to prove conformance, a part falling in the transition region cannot be considered conforming, while, if a costumer wishes to prove non-conformance, a part falling in the transition region cannot be considered non conforming.

\subsection{Evaluating Geometric deviation}

Traditional instruments are not suitable to check geometric tolerances. Geometric tolerances verification is usually performed by means of coordinate metrology. A wide range of coordinate measuring devices exist today on the market, distinguished by their accuracy, working principle (mechanical contact, laser triangulation, image probing,... ), sampling (point-to point, line scanning, area scanning), measuring volume, etc. Regardless of the specific instrument, every coordinate measuring system is based on sampling some points on the surface to inspect. Then, a "substitute geometry" is fitted on these points, and the geometric deviation is evaluated as the maximum deviation from this geometry (see the work by Antony et al. [14] for further details). Because only few points usually define the amplitude of the tolerance zone, only these points are really relevant to evaluate the geometric deviation.

Most coordinate measure system are flexible, being able to sample points on very different surfaces. However, this flexibility makes evaluation of measurement uncertainty difficult since different measurement tasks are characterized

by different uncertainties; hence, a "task specific uncertainty" [15] should be evaluated. In fact, Wilhelm et al. [15] have identified several sources of Coordinate Measuring Machine (CMM) measurement uncertainty such as hardware, workpiece geometry, sampling strategy, fitting and evaluation algorithms, and extrinsic uncertainty sources. In particular several authors have pointed out that the sampling strategy can significantly affect measurement uncertainty (see e.g. Weckenmann et al. [16, 17]; Kruth et al. [18]), in particular when the sample size is small, which may be a typical situation if uncertainty cost has to be opti- 
mized. Because sampling strategy is most often determined by the operator, it is the main leverage to control uncertainty as well. A conprehensive discussion about coordinate measuring devices performance, uncertainty evaluation, and relationship with the conformance or non conformance statement can be found in the recente work by Phyllips [13] in the book by Hocken and Pereira [19].

Methodologies for sampling strategy planning may be grouped into three categories (for further reference on sampling strategy planning, please address to recent works of the authors of the present paper [20,21]). Blind sampling strategy are standard sampling strategies, like those defined in international standards $[22,23,24,25]$, which do not require any knowledge of the surface to inspect, apart from nominal geometry. Adaptive strategies $[26,27]$ try to "adapt" the strategy itself to the actual surface, that is, they sample an initial set of points, and then sequentially choose the next sampling points based on the knowledge of the already sampled points. Finally, manufacturing based sampling strategies are strategies developed for parts manufactured by a specific process [28].

Manufacturing based sampling strategies originate from the observation that sampling strategy uncertainty contribution and the actual part geometric deviation tend to be closely interrelated $[15,18,29]$. If geometric tolerance definitions given by the ISO 1101 standard are followed, only those zones of the surface which deviate the most from the design nominal geometry define the geometric deviation (worst case scenario). There is a strong interrelations between sampling strategy, measurement point layout and capability to identify part out-of-tolerance ("anomalous") zones caused by part geometric deviation patterns that directly affect measurement uncertainty. There is an extensive literature related to development of a generic methods for optimization of measurement point layouts $[30,31,32,33]$. However, it has also been observed that anomalous zones of the part profile/surface tend to be the same throughout the production. It may therefore be stated that the part presents a process inherent error signature - a "manufacturing signature".

Ceglarek et al. [34, 35] developed methods to model part variation patterns 
of pre-assembled components to compensate dimensional variability caused by upstream manufacturing processes. In recent years several studies have suggested that the interaction between sampling strategy and manufacturing process error signature can be analyzed in order to generate very effective sampling strategies (e.g. Summerhayes et al. [36], Killmayer and Babu [37], or Colosimo et al. [28]). However, the criteria adopted for the definition of the optimal strategy are heuristic, and lack an explicit uncertainty evaluation.

\subsection{Paper aims}

In this paper the applicability of an economic framework to the optimization of the sampling strategy is pointed out. A cost function depending on the sampling strategy (i.e. number and location of sampling points) will be reviewed [21]. To complete the framework, two methodologies will be proposed for the evaluation of the measurement uncertainty, whose knowledge is required for the estimate of the cost: the first one experimental, based on the acquisition of data on calibrated workpieces, the second one based on simulation. The two methodologies are based on current state of the art in uncertainty evaluation, and go beyond it by explicitly considering the sampling strategy and its interaction with the manufacturing signature. By optimizing the cost function, while simultaneously correctly evaluating the uncertainty, an optimal sampling strategy can be defined: the best trade-off between measurement costs and measurement uncertainty can be found. An heuristic algorithm will be adopted to optimize the function. Finally, this approach is expected to efficiently interact with manufacturing based sampling strategies. Therefore, it will be shown that the inclusion of the interaction between the sampling strategy and the manufacturing signature in the uncertainty evaluation allows far lower costs.

Case studies will be proposed, involving roundness and parallelism tolerances. 


\section{Economic framework for the evaluation of inspection cost}

The authors of the present work have recently proposed a paper [21] in which an economic framework is presented which allows the evaluation of the inspection cost. In that work the applicability of the framework has been discussed with reference to an early design stage. Here mathematical details of the framework are reported to allow the understanding of its applicability to strategy planning; for a more complete discussion of the methodology the reader is addressed to the paper just cited.

Consider the general case in which $N$ parts have to be inspected; for every part, $T$ geometric tolerances have been defined, and $S$ geometric features are involved. In general, inspection cost is the sum of two costs, one cost $C_{M}$ deriving from performing the measurement, the other $\operatorname{cost} C_{E}$ originating from inspection errors (of both type A and B), that is

$$
C_{I}=C_{M}+C_{E}
$$

\subsection{Measurement $\operatorname{cost} C_{M}$}

$C_{M}$ is itself the sum from two cost: a cost which depends on the measurement strategy $C_{M M}$, and a cost which is independent of it $C_{M F}$. The latter depends mainly on the selected measuring instrument, so in general it is not particularly relevant if sampling strategy planning is the aim.

$C_{M F}$ depends essentially on the chosen measuring instrument. It originates from planning the measurement strategy, and setting up every single part on the measuring instrument for inspecting it. In general, these costs can be considered fixed, and are relevant only if the measuring instrument has to be selected. The only contribution to $C_{M F}$ related to sampling strategy planning is the cost for planning the sampling strategy. In fact, planning a manufacturing based strategy is in general more expensive that planning a blind or adaptive strategy, because costs for acquiring information on manufacturing signature have to be borne. 
In general $C_{M M}$ is proportional to the time required to perform the measurement. To evaluate this time, two different categories of instruments should be considered, i.e. "discrete measuring" instruments, and "continuous measuring" instruments.

Discrete measuring instruments group those instruments whose probing sensor is activated a discrete number of times to measure the feature, each activation requiring a fixed time to sample one or more points. In this case, the measurement cost depends essentially on the number of activations of the sensor, that is

$$
C_{M M}=N c_{m} t_{m} \simeq N c_{m} t_{a} \sum_{j=1}^{S} n_{a j}=N c_{a} \sum_{j=1}^{S} n_{a j}
$$

where $c_{m}$ is the hourly cost of the measuring system, $t_{a}$ is the time required by a single activation of the sensor, $n_{a j}$ is the number of activations required to measure the $j^{\text {th }}$ feature, and $c_{a}$ is the cost of a single activation.

In contrast, in a continuous measuring instrument probings happen with a given frequency, while the sensor moves on the feature to measure. The measurement time for scanning instruments depends on the overall scan length divided by the scan speed:

$$
C_{M M}=N c_{m} t_{m} \simeq N c_{m} \sum_{j=1}^{S} \frac{l_{j}}{v_{j}}=N c_{m} \sum_{j=1}^{S} \frac{\delta_{j} n_{p j}}{v_{j}}
$$

where $l_{j}$ is the length of the scan for the $j^{\text {th }}$ feature, $v_{j}$ is the speed at which this scan is performed, $\delta_{j}$ is the spatial sampling period, and $n_{p j}$ is the number of points scanned on feature $j$.

\subsection{Inspection Error $\operatorname{cost} C_{E}$}

Evaluation of $C_{E}$ is more complicated. Define $f_{A i}\left(f_{B i}\right)$ the event "a type A (B) error is committed when checking tolerance $i$ ". Suppose that the criterion for conformance testing is applied, as suggested in the ISO 14253-1 standard (the solution is similar if the non conformance test is applied). In general, the 
probabilities that a type A or B error happen are

$$
\begin{aligned}
& P\left(f_{A i}\right)=P\left(y_{i} \geq S L_{i}-U_{i} \mid x_{i} \leq S L_{i}\right) \\
& P\left(f_{B i}\right)=P\left(y_{i} \leq S L_{i}-U_{i} \mid x_{i} \geq S L_{i}\right)
\end{aligned}
$$

where $y_{i}$ is the measurement result for the geometric deviation $i, x_{i}$ is the real value of the geometric deviation $i$, and $S L_{i}$ and $U_{i}$ are respectively the specification limit and the extended uncertainty for tolerance $i$. Both $x_{i}$ and $y_{i}$ are random variables, whose statistical distribution should be known given manufacturing process and measuring instrument. Of course, $U_{i}$ is affected by the measurement procedure and instrument, thus justifying the search for a trade of between $C_{M}$ and $C_{E}$.

Bearing in mind this definition for the inspection error probability, it is possible to express the overall cost of type A errors $C_{E A}$ :

$$
C_{E A}=N c_{E A} P\left(\bigcup_{i=1}^{T} f_{A i}\right)
$$

where $c_{E A}$ is the cost for declaring non-conforming a conforming part. $P\left(\bigcup_{i=1}^{T} f_{A i}\right)$ represents the probability that a type A error is committed while verifying any of the $T$ tolerances.

The evaluation of the cost related to type B errors $C_{E B}$ is similar, i.e.

$$
C_{E B}=N c_{E B} P\left(\bigcup_{i=1}^{T} f_{B i}\right)
$$

where $c_{E B}$ is the cost of stating conforming a non conforming part.

Even if computing these probabilities is complicated, in most production processes only few characteristics are really critical. If six sigma condition is met, and if the uncertainty is not large, probabilities in Eq. (4) will be small. Therefore, if critical tolerances can be identified [38], Eq. (5) and (6) can be reasonably solved.

Please note that, in general, $P\left(f_{B i}\right)$ is small if a conformance test is performed, and vice versa $P\left(f_{A i}\right)$ is small in case of non conformance test [2]. This 
can lead to a simplified expression of these probabilities, that is

$$
\begin{aligned}
& P\left(f_{A i}\right)=P\left(S L_{i}-U_{i} \leq x_{i} \leq S L_{i}\right) \\
& P\left(f_{B i}\right)=0
\end{aligned}
$$

when conformance test is performed, and

$$
\begin{aligned}
& P\left(f_{B i}\right)=P\left(S L_{i} \leq x_{i} \leq S L_{i}+U_{i}\right) \\
& P\left(f_{A i}\right)=0
\end{aligned}
$$

when the non-conformance test is performed. These probabilities may be interpreted as the fraction of wrongly rejected (conformance test, see Fig. 2) or accepted (non-conformance test) parts. This formulation is in general easier to manage.

\section{Measurement Uncertainty evaluation}

The economic model described in $\S 2$ can, in general, evaluate the inspection cost for geometric tolerances. Eq. (4) clearly shows the importance of uncertainty in this evaluation. If the optimal sampling strategy is the aim, then the uncertainty evaluation procedure has to clearly consider the contribution of the sampling strategy (sampling points pattern and number). Wilhelm et al. [15] have identified six ways for evaluating Coordinate Measuring Systems (CMS) measurement uncertainty: sensitivity analysis, expert judgment, use of calibrated objects, computer simulation, statistical estimation from measurement history, and hybrid methods. Among these methodologies, expert judgment and sensitivity analysis are not effective to consider the influence of the sampling points pattern. Statistical estimation from measurement history requires historical data to be available, which is usually not possible in strategy design phase. And hybrid methods are just a derivation of the other methodologies. Therefore, two procedures will be proposed, one based on the use of calibrated artifacts identical or similar to parts that will be manufactured, and one on simulation. A discussion an the evaluation of uncertainty for coordinate measuring devices has been proposed by Phillips [13]. 


\subsection{Calibrated artifacts based procedure}

The ISO/TS 15530-3 [39] standard proposes a method to evaluate CMM uncertainty. However, the principle can be extended from CMMs to any CMSs. According to the proposed procedure, $n$ repeated measurements (the ISO/TS 15530-3 standard suggests $n \geq 20$ ) are carried out on one or more calibrated workpieces, then from these results terms $u_{\mathrm{cal}}, u_{\mathrm{p}}, u_{\mathrm{W}}$, and $u_{\mathrm{b}}$ are estimated, and $U$ is evaluated as

$$
U=k \sqrt{u_{\mathrm{cal}}^{2}+u_{\mathrm{p}}^{2}+u_{\mathrm{W}}^{2}+u_{\mathrm{b}}^{2}}
$$

where $k$ is the coverage factor [1], $u_{\text {cal }}$ is the standard calibration uncertainty of the artifact(s) adopted, $u_{\mathrm{p}}$ is the uncertainty contribution due to the measuring system adopted, $u_{\mathrm{W}}$ is the uncertainty contribution related to the production variability (e.g. non perfectly constant thermal expansion coefficient of the material, or the adoption of a calibrated artifact that significantly differs from manufactured parts), and $u_{\mathrm{b}}$ is the uncertainty due the compensation of the bias, which can also be estimated from the $n$ measurement repetitions. $u_{\mathrm{p}}$ can be experimentally evaluated, in the case only an artifact is adopted, as

$$
u_{p}=\sqrt{\frac{1}{n-1} \sum_{i=1}^{n}\left(y_{i}-\frac{1}{n} \sum_{i=1}^{n} y_{i}\right)}
$$

where $x_{\text {cal }}$ is the calibrated value of the geometric deviation of the artifact.

If a blind or adaptive sampling strategy is being planned, then Eq. (9) and (10) are sufficient to apply the economic framework. It has to be pointed out that only the sample size will be defined for blind strategies, because the sampling points pattern is either a priori established, or adaptively chosen for every part. But if a manufacturing based strategy has to be planned, then considering only a single artifact cannot encompass the part to part variability of the production, and any sampling point pattern defined this way will tend to reflect the actual geometric deviation of the adopted calibrated artifact. Therefore, a slight modification of these formulas is proposed, so that more than one workpiece may be used. In particular, to estimate $u_{\mathrm{p}}$, a pooled standard deviation 
should be used:

$$
u_{\mathrm{p}}=\sqrt{\frac{\sum_{j=1}^{m} \sum_{i=1}^{r_{m}}\left(y_{i, j}-\frac{1}{n} \sum_{k=1}^{n} y_{k, j}\right)}{m\left(r_{m}-1\right)}}
$$

where $m$ is the number of artifacts adopted, and $r_{m}$ is the number of measurement repetitions per calibrated artifact. It is supposed that each calibrated workpiece is measured the same number of times; in general, it can be suggested $r_{m} \geq 10$.

Substituting Eq. (11) in Eq. (9) the required evaluation of $U$ results.

A final note on $u_{\mathrm{W}}$ : ISO $15530-3$ introduces it to consider the variability of the production; however, if more than one calibrated workpiece is measured, then the term $u_{\mathrm{p}}$ should contain this uncertainty contribution, and $u_{\mathrm{W}}=0$.

\subsection{Simulation based procedure}

Computer simulation [40] is probably the most suitable method for evaluating a "task specific uncertainty" for coordinate measurement systems, having the required flexibility, and not being subjective like expert judgment. This subject has been particularly well described for CMMs, but can be extended to any coordinate measuring system. A software simulating the behavior of a CMM, allowing uncertainty evaluations, is commonly known as "virtual CMM".

A classical virtual CMM $[15,41]$ is based on simulation of ideal (but not necessarily perfect, e.g. they can contain some undulations) geometric features, for which the geometric deviation is known. A measurement error is simulated according to a model of the real behavior of the CMM for which the uncertainty is being evaluated, and added to the ideal feature. Measurement uncertainty is evaluated by comparing geometric deviations evaluated on the "perturbed" features and the known geometric deviations of ideal features. The overall methodology may be regarded as a Montecarlo simulation of ideal profiles and sampling errors, from which a Montecarlo simulation of geometric deviation evaluation error $x-y$ is derived.

Various error sources should be considered in the error simulations, including measurement strategy, environmental conditions, CMM volumetric errors, and 
so on. A complete list of these sources may be found in the ISO/TS 15530-4 standard [42]. This standard deals with the problem of validating virtual CMM models, proposing four methodologies for the validation, one of which has been adopted to validate the virtual CMM developed for producing results in this paper, as it will be discussed later in this paragraph.

Several methodologies have been proposed to extrapolate uncertainty from simulation results. In this paper, the approach proposed by Schwenke et al. [43] has been adopted. This approach does not allow to explicitly calculate a standard uncertainty $u$, but only an expanded uncertainty $U$ characterized by some coverage probability $p[1]$. Suppose a Montecarlo simulation of several (thousands) geometric deviation evaluation errors $x-y$ is available. From this data a Montecarlo evaluation of the statistical distribution of $x-y$ is derived. Let's define $G(x-y)$ the empirical cumulative distribution of $x-y$. Therefore an evaluation of the expanded uncertainty characterized by the coverage probability $p$ may be obtained as (Fig. 3)

$$
G(U)-G(-U)=p
$$

Please note that the resulting evaluation of $U$ is coherent with the definition of coverage probability, and that any uncorrected bias is considered [43].

To simulate geometric deviations, an approach based on results proposed by Van Dorp et al. [44] has been implemented. The developed model is based on the frequency content of the error signal, and may be applied to a "Zeiss Prismo" CMM. At present the virtual CMM model implemented takes into account only the geometric deviation of the CMM and the adopted sampling strategy as uncertainty sources.

However, in order to validate the Virtual CMM, the approach proposed in Annex C.2 of ISO/TS 15530-4 standard has been chosen. The virtual CMM has generated an uncertainty evaluation for roundness measurement of a calibrated plug gauge, ensuring a 95\% coverage probability, and simulating one thousand measurement repetitions; the uncertainty statement is supposed to be valid for the "Zeiss Prismo" CMM available at Politecnico di Milano, where this work 
has been developed. Then the calibrated plug gauge has been measured one hundred times. The formula [42]

$$
\left|y_{\text {cal }}-y\right| \leqslant \sqrt{U_{\text {cal }}^{2}+U^{2}}
$$

has been applied for proving plausibility of the uncertainty evaluation (where $y_{\text {cal }}$ is the calibrated roundness of the plug gauge, and $U_{\text {cal }}$ is the calibration expanded uncertainty with a coverage probability of about 95\%). 97 of 100 measurement repetitions satisfied Eq. (13), thus proving virtual CMM uncertainty evaluation plausibility.

The standard model of virtual CMM which simulates "standard" geometric feature is adequate if an optimal blind or adaptive strategy is to be defined. If a manufacturing based strategy has to be planned, the virtual CMM must take into account the interaction between the actual geometric deviation and the sampling strategy when evaluating the measurement uncertainty. This is not hard to obtain with a Montecarlo simulation based virtual CMM: it is sufficient

to simulate nominal profiles according to a model of the actual signature. If the simulated ideal profiles are generated according to some real signature model instead of being features perfect or characterized by "standard" error components, like undulations, then the uncertainty evaluation will implicitly consider the signature. It will suffice to simulate, according to the manufacturing signature model, a sufficient number of profiles on a dense set of sampling points: geometric deviation evaluated on this profiles is the simulated real geometric deviation $x$. In order to obtain the simulated measurement result $y$ points related to the considered sampling strategy will be extracted, and a random measurement error will be added to each point: the error evaluated on this "perturbed" profile is $y$. Evaluation of error $x-y$ follows.

\section{Defining an optimal sampling strategy}

Now the framework for defining an optimal sampling strategy is complete. Just an algorithm to solve the problem is missing. 
However, the solution of the problem is not straightforward. The definition of the absolutely optimal sampling strategy requires that any sampling strategy is considered, but infinite sampling patterns exist. Theoretically a simulation approach should be able to solve the problem, but at very high computational cost; experimental approach is absolutely unable to manage any situation, because the surface is known only on a discrete number of sampling points. Therefore, to simplify the problem, only a finite number of locations on the surface(s) to inspect, instead of any location, will be considered as candidate sampling points. Such locations will be sampled on every calibrated artifact, in the calibrated artifact based approach to uncertainty evaluation, or simulated, in the simulation based approach. This way, the number of possible sampling strategies is still $\sum_{k=1}^{n_{p}} \frac{n_{p} !}{k !\left(n_{p}-k\right) !}$ (where $n_{p}$ is the number of candidate sampling points), which is very large if $n_{p}>1000$ as usual. Therefore, given the discrete nature of the problem, an optimization algorithm, like a genetic algorithm [45] or a simulated annealing algorithm [46], is suitable for the task - even if the proposed solution will not be necessarily the global optimum, this kind of algorithms may reduce the number of strategies to test, so that a sub-optimal strategy may be calculated in a reasonable time.

\subsection{Artifact based approach vs. Simulation based approach}

Having introduced how to plan an optimal sampling strategy, some ambiguity remains on the choice between a calibrated artifact based approach or a simulation based approach to the evaluation of the uncertainty. Either method has peculiar advantages.

Artifact based method is, in general, easier to apply. It just requires that one or more calibrated artifacts, which in this case are standard parts produced by the process or ad hoc produced specimen, to be acquired and repeatedly measured on an adequately large number of sampling locations. No particular skill is required in doing this, nor any particular difficulty can be envisaged. If artifacts are manufactured by the actual process, even a manufacturing based strategy can be easily defined, which can lead to very low uncertainty [47, 48]. 
But a simulation based approach can, in general, be less expensive. In fact, no material reference artifact is required to apply this methodology. Application is, in general, a bit more difficult, and more care must be taken in setting simulation parameters. However, this approach allows a real task specific uncertainty evaluation even if only few parts are manufactured. In general, it is possible to afford the cost for developing calibrated artifacts only in medium or

large scale production, so the first approach is applicable only in a limited series of situations.

If a manufacturing based strategy is being developed, then simulation approach has another advantage. In general, manufacturing based strategies become very unreliable if the manufacturing process is not stable, which causes the manufacturing signature to change overtime. The development of a manufacturing based strategy with a simulation based uncertainty evaluation requires a model of the signature to be known. In recent years several signature based monitoring techniques have been proposed $[49,50,51,52]$ which require a signature model, and which are very effective in process monitoring. Therefore, the choice of a simulation and manufacturing based optimal sampling strategy efficiently couples with reactive monitoring techniques.

\section{Case studies}

Three case studies are dealt with: two dealing with roundness, with either an artifact or simulation based approach, and one dealing with plane parallelism, dealt with both an artifact and a simulation based approach. In every case study, a conformance test will be considered, and the simplified formula for the calculation of the probability, i.e. Eq. 7, will be considered.

\subsection{Roundness - artifact based}

This case study [47] involves the roundness of ten turned cylindrical specimens in titanium alloy Ti-6Al-4V grade 5. Geometry, cutting parameters, and costs as follows: 
- Turned length: $47 \mathrm{~mm}$

- Final diameter: $16.8 \mathrm{~mm}$

- Depth of cut $a_{\mathrm{p}}: 1.2 \mathrm{~mm}$

- Feed $f: 0.14 \mathrm{~mm} / \mathrm{rev}$

- Cutting speed $v_{\mathrm{c}}: 65 \mathrm{~m} / \mathrm{min}$

- Production cost, considered as cost of the type A error $c_{E A}$, for a single specimen: $23.6 €$

A single roundness profile was then sampled on each specimen, $2 \mathrm{~mm}$ from the edge the farthest from the self-centering chuck. A "Zeiss Prismo VAST HTG" CMM performed measurements, whose main characteristics follow:

- Maximum permissible error: $P_{\mathrm{FTU}, \mathrm{MPE}}=2 \mu \mathrm{m}$

- Hourly cost: $65 € / \mathrm{h}$

- Time to sample a single point: $1.5 \mathrm{~s}$

Specimens were sampled on 1250 points (a point every about $0.005 \mathrm{rad}$ ). The typical roundness error is shown in Fig. 4.

The presence of a signature characterized by the superimposition of a bilobed and a tri-lobed shape is apparent. A reasonable supposition is that this error is due to defects in spindle rotation.

The roundness of each specimen has been calibrated, with $u_{\text {cal }}=1.5 \mu \mathrm{m}$. An Anderson-Darling normality test for these ten calibrated values suggests that there is not a statistical evidence to refuse the normality of the out of roundness distribution, so it is supposed that $x \sim N(18.7,2.8)$ (mean and standard deviation expressed in $[\mu \mathrm{m}]$ ), as estimated from data. A roundness tolerance of $0.023 \mathrm{~mm}$ was defined on the measured profile. Then, each specimen has been measured ten times, on 1250 points.

The application of a simulated annealing algorithm has then lead to a manufacturing based sampling strategy. A seven points strategy (Fig. 5) resulted 
optimal, whose inspection cost was $6.6 €$; Fig. 5 shows the strong link between the optimal sampling strategy and the signature, too. This value may be compared to the inspection cost with a blind uniformly spaced strategy, which is $8.6 €$. Being the sample size the same, cost reduction depends only on the optimization of the sampling strategy for the particular manufacturing signature: expanded uncertainty for the uniformly spaced strategy is $3.7 \mu \mathrm{m}$, while $U$ for the optimized strategy is $3.1 \mu \mathrm{m}$ ( $k=2$ for both estimates, as suggested by both ISO 14253-1 and ISO/TS 15530-3). Moreover, the optimal cost for a uniform strategy is $8.2 €$ (corresponding to fifteen points). For further explanation, consider Fig. 6, which shows the measurement uncertainty as the sample size varies for both blind and manufacturing based sampling strategies. It is apparent that the manufacturing based sampling strategy outperforms the blind sampling strategy regardless of the sampling size. A similar result is shown by Fig. 7 , which shows the behavior of inspection cost $C_{I}$. In figure 7 it is also apparent that, for large sample sizes, the cost difference between the blind and the manufacturing based strategy is very small. This is due to the general reduction of $U$. If $U$ is sufficiently small, then $P\left(L S_{i}-U_{i} \leq x_{i} \leq L S_{i}\right)$ is small, too, so only sampling points number affects the inspection cost according to Eq. (1), (5), and (7).

\subsection{Roundness - simulation based}

This case study [53] is based on the manufacturing signature model proposed by Colosimo et al. [54]. This model describes the signature of roundness profiles obtained by turning C20 steel bars. Geometry, cutting parameters, and costs as follows:

- Turned length: $60 \mathrm{~mm}$

- Final diameter: $26 \mathrm{~mm}$

- Depth of cut $a_{\mathrm{p}}: 5 \mathrm{~mm}$

- Feed $f: 0.2 \mathrm{~mm} / \mathrm{rev}$ 
- Cutting speed $v_{\mathrm{c}}: 163 \mathrm{~m} / \mathrm{min}$

- Production cost, considered as cost of the type A error $c_{E A}$, for a single specimen: $10 €$

One hundred specimens were manufactured, and measured on 748 evenly spaced points by means of the same CMM described in $§ 5.1$. From this data, a "Spatially Autocorrelated Regression" model of the second order SARX(2) [55] signature model has been identified. An instance of the geometric deviation generated according to this manufacturing signature model is depicted in Fig. 8. Similarly, Fig. 9 shows the average profile. Both figures show the local form deviation as a function of the angle $\theta$.

By means of this signature model, one thousand "ideal" profiles have been simulated, the roundness error of which has been taken as reference value. Roundness reference values have shown a normal distribution of geometric deviation, so it is assumed that $x \sim N(14.6,1.4)$, with mean and standard deviation expressed in $[\mu \mathrm{m}]$. Moreover, a roundness tolerance of $25 \mu \mathrm{m}$ is considered for these profiles.

A simulated sampling error has been added to the simulated profiles, adopting the VCMM approach described in $§ 3.2$, thus generating one thousand "perturbed" profiles. Finally, a simulated annealing algorithm has been applied to this data, in order to optimize the sampling strategy as described in $\S 4$. An optimal sampling strategy constituted by 14 points has been identified (Fig. 9 ). As expected sampling points are concentrated where roundness deviation is greatest, so that the bias in measurement of geometric deviation is minimized.

Finally, Fig. 10 and 11 portrait respectively the behavior of uncertainty and inspection cost as the sample size changes. Considerations similar to those drawn for the titanium specimens in $§ 5.1$ follow.

\subsection{Parallelism}

The case studies proposed so far regard roundness, i.e. a form tolerance.

In a form tolerance only a geometrical feature is involved (no datum feature is 
defined). Therefore, sampling regards only the toleranced feature, and the proposed optimization can be applied easily. However, the proposed methodology is in principle applicable to any geometric tolerance. To prove this consider as case study the parallelism defined in point (e) of Table 3 in the ISO 10791-7 standard [56]. This case study will be solved by both an artifact [48] and a simulation [57] based approach. Ten parts were contour milled [58] adopting the following cutting parameters:

- Mill diameter: $40 \mathrm{~mm}$

- Depth of cut $a_{\mathrm{p}}: 6 \mathrm{~mm}$

- Engagement $a_{\mathrm{e}}: 0.2 \mathrm{~mm}$

- Feed per tooth $f_{\mathrm{z}}: 0.15 \mathrm{~mm} / \mathrm{rev}$

- Cutting speed $v_{\mathrm{c}}: 350 \mathrm{~m} / \mathrm{min}$

- Number of teeth $Z_{\mathrm{n}}: 4$

- Production cost, considered as cost of the type $\mathrm{A}$ error $c_{E A}$, for a single specimen: $40 €$

and a $0.045 \mathrm{~mm}$ parallelism tolerance was defined. Then tolerances and datum features were densely sampled by means of the same CMM described in $§ 5.1$. For this high density sampling a uniformly spaced sampling strategy, with a point density of 1 point $/ \mathrm{mm}^{2}$, was adopted; a total of 3720 points were sampled on each part (1395 on the datum feature, and 2325 on the toleranced feature). Measurement was repeated ten times for each part. Finally, every part was calibrated with a calibration standard uncertainty $u_{\text {cal }}=0.001 \mathrm{~mm}$. From calibrated parallelism errors, it was ascertained that parallelism for these parts was distributed according to a $N(0.041,0.0017)$ Gaussian statistical distribution (mean and standard uncertainty expressed in $[\mathrm{mm}]$ ). The average surface of the ten toleranced surfaces is plotted in Fig. 12: it is apparent that the surface presents a sawtooth profile, which is due to the multiple cutting steps required 
for the overall machining, and a trend along the $z$ axis, which depend on mill inflection, and generates a lack of parallelism between the average planes of the toleranced and datum features.

The data gathered as just described form the base to apply the artifact based approach to the optimization of the sampling strategy. To apply a simulation based approach, based on these data a numerical model has been proposed [58] which simultaneously describes both the toleranced and datum features. The model has been adopted to simulate one thousand surfaces, and the related measurement error.

Finally, a simulated annealing algorithm was applied in both the artifact and the simulation based approach in order to select an optimal sampling strategy. Please note a parallelism is being considered, so sample size sums both the points sampled on the toleranced feature and on the corresponding datum feature, and, throughout the optimization process, sampling points are left free to "migrate" from datum to toleranced feature and vice versa. Optimal strategy was compared to a standard Hammersley strategy [59], which is considered as reference blind strategy. The final results are reported in Fig. 13 and 14 for the artifact based approach, and in Fig. 15 and 16 for the simulation based approach. These graphs confirm what has already been stated for roundness inspection in $\S 5.1$ and $\S 5.2$. A couple more considerations can be added. First, the selected sample size tends to be larger than in the case of roundness inspection. The spread between blind strategies cost and manufacturing based strategies cost tends to be larger, too. This can be ascribed to the difficulty of blind strategies in completely and densely sampling a 2D surfaces, difficulty which is less significant in the case of $1 \mathrm{D}$ profiles. Second, optimal cost and sample sizes are similar in the cases of simulation and artifact based optimization approaches. This indicates a general coherence between the two approaches. 


\section{Conclusions}

Inspection cost is often neglected when evaluating the manufacturing cost. However, it can be a very significant contributor. In this work, a model for the evaluation of inspection cost has been proposed. It has been clearly shown how, according to this model, the inspection cost is strongly influenced by the measurement uncertainty of the inspection system adopted. Then, two approaches for evaluating the uncertainty in the case of coordinate measurement have been introduced. Based on this approaches together with the cost model it is possible to choose the optimal sample size. Moreover, if some knowledge about the manufacturing signature is available, it is also possible to define an optimal sampling points pattern, thus defining the most cost effective sampling strategy. Few case studies have been proposed, showing that the approach can lead to significant savings in manufacturing, as Kunzmann et al. have suggested [2].

Future developments include a deep analysis of the effect of correlations between tolerances on the inspection cost, in particular in the case in which more than a tolerance is defined on a single part, and the same geometric feature is shared by differing tolerances, the extension of the procedure to the case in which continuous measuring and area scanning systems are involved, and the development and consideration in the result evaluation of adaptive sampling strategies.

\section{Acknowledgments}

Financial support to this work has been provided by Italian Ministry of Education, University and Research, as part of the project PRIN2008 (prot. 200853ZT3Z).

[1] ISO/IEC, ISO/IEC GUIDE 99:2007(E/F): International vocabulary of basic and general terms in metrology (VIM) (2007).

[2] H. Kunzmann, T. Pfeifer, R. Schmitt, H. Schwenke, A. Weckenmann, Productive metrology - adding value to manufacture, CIRP Annals - 
Manufacturing Technology 54 (2) (2005) 155-168. doi:10.1016/S00078506(07)60024-9.

[3] ISO/IEC, ISO/IEC GUIDE 98-3: Uncertainty of measurement - Part 3: Guide to the expression of uncertainty in measurement (GUM:1995) (2008).

[4] International Organization for Standardization, ISO 1101: Geometrical product specifications (GPS) - tolerances of form, orientation, location and run out (2012).

[5] The American Society of Mechanical Engineers, ASME Y14.5M: Dimensioning and tolerancing (2009).

[6] The American Society of Mechanical Engineers, ASME B89.7.3.1: Guidelines for decision rules: Considering measurement uncertainty in determining conformance to specifications (2001).

[7] The American Society of Mechanical Engineers, ASME B89.7.3.2: Guidelines for the evaluation of dimensional measurement uncertainty (2007).

[8] The American Society of Mechanical Engineers, ASME B89.7.3.3: Guidelines for assessing the reliability of dimensional measurement uncertainty statements (2002).

[9] International Organization for Standardization, ISO 14253-1: Geometrical product specifications (GPS) - inspection by measurement of workpieces and measuring equipment - part 1: Decision rules for proving conformance or nonconformance with specifications (1998).

[10] International Organization for Standardization, ISO/TS 14253-2: Geometrical product specifications (GPS) - inspection by measurement of workpieces and measuring equipment - part 2: Guide to the estimation of uncertainty in GPS measurement, in calibration of measuring equipment and in product verification (1999). 
[11] International Organization for Standardization, ISO/TS 14253-3: Geometrical product specifications (GPS) - inspection by measurement of workpieces and measuring equipment - part 3: Guidelines for achieving agreements on measurement uncertainty statements (2002).

[12] S. D. Phillips, Measurement uncertainty and traceability issues in national and international measurements, in: International Dimensional Metrology Workshop, Nashville, TN (USA), 2003.

[13] S. D. Phillips, Performance Evaluation, in: Hocken and Pereira [19], Ch. 9, pp. $183-272$.

[14] G. T. Anthony, H. M. Anthony, B. Bittner, B. P. Butler, M. G. Cox, R. Drieschner, R. Elligsen, A. B. Forbes, H. Gross, S. A. Hannaby, P. M. Harris, J. Kok, Reference software for finding chebyshev best-fit geometric elements, Precision Engineering 19 (1) (1996) 28 - 36. doi:10.1016/01416359(96)00005-0.

[15] R. G. Wilhelm, R. Hocken, H. Schwenke, Task specific uncertainty in coordinate measurement, CIRP Annals - Manufacturing Technology 50 (2) (2001) 553-563. doi:10.1016/S0007-8506(07)62995-3.

[16] A. Weckenmann, H. Eitzert, M. Garmer, H. Weber, Functionality-oriented evaluation and sampling strategy in coordinate metrology, Precision Engineering 17 (4) (1995) 244-252. doi:10.1016/0141-6359(94)00020-Z.

[17] A. Weckenmann, M. Knauer, H. Kunzmann, The influence of measurement strategy on the uncertainty of CMM-measurements, CIRP Annals - Manufacturing Technology 47 (1) (1998) 451 - 454. doi:10.1016/S00078506(07)62872-8.

[18] J. P. Kruth, N. Van Gestel, P. Bleys, F. Welkenhuyzen, Uncertainty determination for CMMs by monte carlo simulation integrating feature form deviations, CIRP Annals - Manufacturing Technology 58 (1) (2009) 463466. doi:10.1016/j.cirp.2009.03.028. 
[19] R. J. Hocken, P. H. Pereira (Eds.), Coordinate Measuring Machines and Systems, 2nd Edition, CRC Press, Boca Raton, New York, 2012.

[20] G. Moroni, S. Petrò, Coordinate measuring machine measurement planning, in: B. M. Colosimo, N. Senin (Eds.), Geometric Tolerances - Impact on Product Design, Quality Inspection and Statistical Process Monitoring, Springer-Verlag, London, UK, 2011, Ch. 4, pp. 111-158. doi:10.1007/9781-84996-311-4.

[21] G. Moroni, S. Petrò, T. Tolio, Early cost estimation for tolerance verification, CIRP Annals - Manufacturing Technology 60 (1) (2011) 195-198. doi:10.1016/j.cirp.2011.03.010.

[22] International Organization for Standardization, ISO 12180-2: Geometrical Product Specifications (GPS) - Cylindricity - Part 2: Specification operators (Apr. 2011).

[23] International Organization for Standardization, ISO 12181-2: Geometrical Product Specifications (GPS) - Roundness - Part 2: Specification operators (Apr. 2011).

[24] International Organization for Standardization, ISO 12780-2: Geometrical Product Specifications (GPS) - Straightness - Part 2: Specification operators (Apr. 2011).

[25] International Organization for Standardization, ISO/TS 12781-2: Geometrical Product Specifications (GPS) - Flatness - Part 2: Specification operators (Dec. 2003).

[26] R. Edgeworth, R. G. Wilhelm, Adaptive sampling for coordinate metrology, Precision Engineering 23 (3) (1999) 144-154. doi:10.1016/S01416359(99)00004-5.

[27] R. Ascione, G. Moroni, S. Petrò, D. Romano, Adaptive inspection in coordinate metrology based on kriging models, Precision Engineering 37 (1) (2013) 44 - 60. doi:10.1016/j.precisioneng.2012.06.006. 
[28] B. M. Colosimo, G. Moroni, S. Petrò, A tolerance interval based criterion for optimizing discrete point sampling strategies, Precision Engineering 34 (4) (2010) 745 - 754. doi:10.1016/j.precisioneng.2010.04.004.

[29] J. M. Sprauel, J. M. Linares, J. Bachmann, P. Bourdet, Uncertainties in CMM measurements, control of ISO specifications, CIRP Annals Manufacturing Technology 52 (1) (2003) 423-426. doi:10.1016/S00078506(07)60616-7.

[30] A. Khan, D. Ceglarek, J. Ni, Sensor location optimization for fault diagnosis in multi-fixture assembly systems, Journal of Manufacturing Science and Engineering 120 (1998) 781-792. doi:10.1115/1.2830221.

[31] A. Khan, D. Ceglarek, J. Shi, J. Ni, T. Woo, Sensor optimization for fault diagnosis in single fixture systems: a methodology, Journal of Manufacturing Science and Engineering 121 (1999) 109-117. doi:10.1115/1.2830562.

[32] A. Khan, D. Ceglarek, Sensor optimization for fault diagnosis in multifixture assembly systems with distributed sensing, Journal of Manufacturing Science and Engineering 122 (2000) 215-226. doi:10.1115/1.538917.

[33] Y. Ding, P. Kim, D. Ceglarek, J. Jin, Optimal sensor distribution for variation diagnosis in multistation assembly processes, IEEE Transactions on Robotics and Automation 19 (4) (2003) 543-556. doi:10.1109/TRA.2003.814516.

[34] D. Ceglarek, M. Szafarczyk, Multivariate analysis and evaluation of adaptive sheet metal assembly systems, CIRP Annals - Manufacturing Technology 47 (1) (1998) 17-22. doi:10.1016/S0007-8506(07)62776-0.

[35] W. Huang, D. Ceglarek, Mode-based decomposition of part form error by discrete-cosine-transform with implementation to assembly and stamping system with compliant parts, CIRP Annals - Manufacturing Technology 51 (1) (2002) 21-26. doi:10.1016/S0007-8506(07)61457-7. 
[36] K. D. Summerhays, R. P. Henke, J. M. Baldwin, R. M. Cassou, C. W. Brown, Optimizing discrete point sample patterns and measurement data analysis on internal cylindrical surfaces with systematic form deviations, Precision Engineering 26 (1) (2002) 105 - 121. doi:10.1016/S01416359(01)00106-4.

[37] T. Killmaier, A. R. Babu, Genetic approach for automatic detection of form deviations of geometrical features for effective measurement strategy, Precision Engineering 27 (4) (2003) 370-381. doi:10.1016/S0141-6359(03)000436.

[38] F. Fiorentini, G. Moroni, P. Palezzato, Q. Semeraro, Feature selection for an automatic inspection system, in: Proceedings of 24th CIRP International Seminar of Manufacturing Systems, Copenhagen, Denmark, 1992, pp. 199-208.

[39] International Organization for Standardization, ISO/TS 15530-3: Geometrical product specifications (GPS) - coordinate measuring machines (CMM): Technique for determining the uncertainty of measurement - part 3: Use of calibrated workpieces or standards (2004).

[40] ISO/IEC, ISO/IEC GUIDE 98-3/Suppl. 1: Uncertainty of measurement Part 3: Guide to the expression of uncertainty in measurement (GUM:1995) - Supplement 1: Propagation of distributions using a Monte Carlo method (2008).

[41] A. Balsamo, M. Di Ciommo, R. Mugno, B. I. Rebaglia, E. Ricci, R. Grella, Evaluation of CMM uncertainty through monte carlo simulations, CIRP Annals - Manufacturing Technology 48 (1) (1999) 425-428. doi:10.1016/S0007-8506(07)63218-1.

[42] International Organization for Standardization, ISO/TS 15530-4: Geometrical product specifications (GPS) - coordinate measuring machines (CMM): Technique for determining the uncertainty of measurement - part 
4: Evaluating task-specific measurement uncertainty using simulation (Jun. 2008).

[43] H. Schwenke, B. R. L. Siebert, F. Wäldele, H. Kunzmann, Assessment of uncertainties in dimensional metrology by monte carlo simulation: Proposal of a modular and visual software, CIRP Annals - Manufacturing Technology 49 (1) (2000) 395-398. doi:10.1016/S0007-8506(07)62973-4.

[44] B. W. van Dorp, H. Haitjema, F. Delbressine, R. H. Bergmans, P. H. J. Schellekens, Virtual CMM using monte carlo methods based on frequency content of the error signal, in: J. E. Decker, N. Brown (Eds.), Proceedings of SPIE 4401 - Recent Developments in Traceable Dimensional Measurements, Vol. 4401, SPIE, 2001, pp. 158-167. doi:10.1117/12.445616.

[45] M. Kudo, J. Sklansky, Comparison of algorithms that select features for pattern classifiers, Pattern Recognition 33 (1) (2000) 25-41. doi:10.1016/S0031-3203(99)00041-2.

[46] S. Kirkpatrick, C. D. Gelatt, M. P. Vecchi, Optimization by simulated annealing, Science 220 (4598) (1983) 671-680. doi:10.1126/science.220.4598.671.

[47] G. Moroni, S. Petrò, CMM measurement uncertainty reduction via sampling strategy optimization, in: Proceedings of the 9th Biennial ASME Conference on Engineering Systems Design and Analysis, Haifa, Israel, 2008, pp. CD-ROM.

[48] D. Ceglarek, G. Moroni, S. Petrò, Manufacturing process error signature and CMM uncertainty costs, in: Proceedings of the 25th Annual Meeting of the American Society for Precision Engineering, Atlanta, GA, USA, 2010, pp. 321-324.

[49] W. H. Woodall, D. J. Spitzner, D. C. Montgomery, S. Gupta, Using control charts to monitor process and product quality profiles, Journal of Quality Technology 36 (3) (2004) 309-320. 
[50] W. Jensen, J. B. Birch, W. H. Woodall, Monitoring correlation within linear profiles using mixed models, Journal of Quality Technology 40 (2) (2008) 167-183.

[51] B. M. Colosimo, M. Pacella, A comparison study of control charts for statistical monitoring of functional data, International Journal of Production Reserch 47 (1) (2009) 1-27. doi:10.1080/00207540802662888.

[52] B. M. Colosimo, F. Mammarella, S. Petrò, Quality control of manufactured surfaces, in: H.-J. Lenz, P.-T. Wilrich, W. Schmid (Eds.), Frontiers in Statistical Quality Control 9, Physica-Verlag HD, Berlin, Germany, 2010, pp. 55-70. doi:10.1007/978-3-7908-2380-6.

[53] G. Moroni, S. Petrò, Virtual CMM based sampling strategy optimization, in: M. Giordano, L. Mathieu, F. Villeneuve (Eds.), Product LifeCycle Management: Geometric Variations, Wiley-ISTE, London, UK, 2010, Ch. 22, pp. 385-404.

[54] B. M. Colosimo, Q. Semeraro, M. Pacella, Statistical process control for geometric specifications: On the monitoring of roundness profiles, Journal of Quality Technology 40 (1) (2008) 1-18.

[55] N. A. C. Cressie, Statistics for Spatial Data, 1st Edition, WileyInterscience, New York, 1993.

[56] International Organization for Standardization, ISO 10791-7: Test conditions for machining centres - part 7: Accuracy of a finished test piece (1998).

[57] G. Moroni, S. Petrò, Optimal sampling strategy for orientation tolerance verification, in: Proceedings of the 7th CIRP International Conference on Intelligent Computation in Manufacturing Engineering, Capri, Italy, 2010.

[58] S. Petrò, G. Moroni, Modeling of surfaces subject to orientation tolerances, in: Proceedings of the ASME 2010 10th Biennial Conference on Engineering Systems Design and Analysis, Istanbul, Turkey, 2010, pp. CD-ROM. 
[59] G. Lee, J. Mou, Y. Shen, Sampling strategy design for dimensional measurement of geometric features using coordinate measuring machine, International Journal of Machine Tools and Manufacture 37 (7) (1997) 917-934. doi:10.1016/S0890-6955(96)00096-X.

\section{Figure Captions}

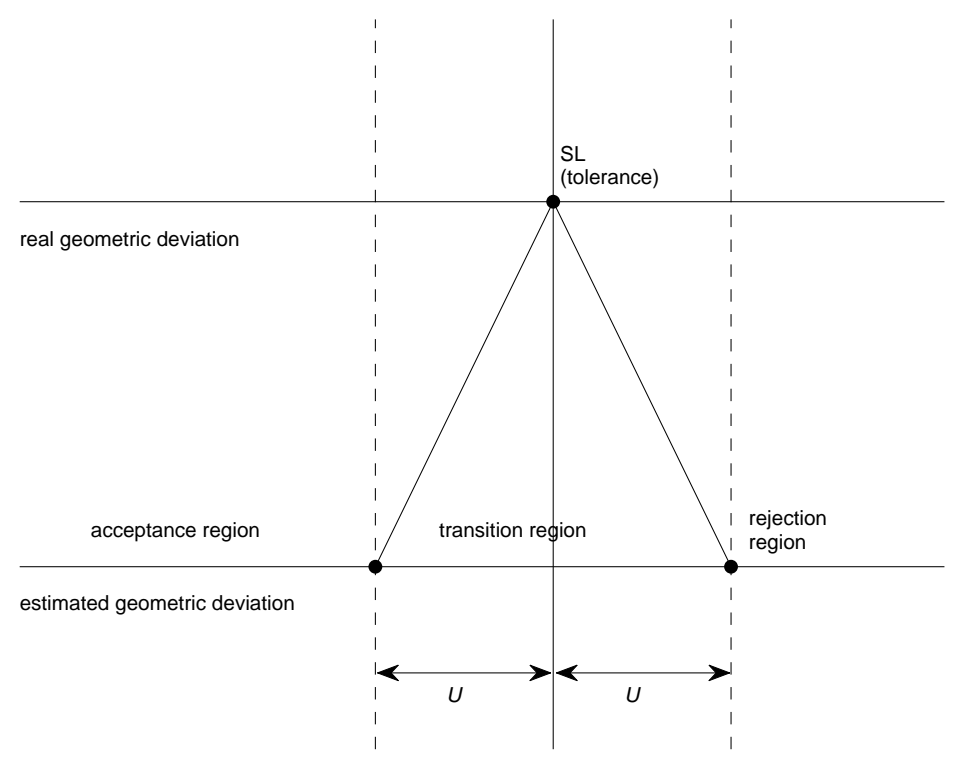

Figure 1: Regions according to the ISO 14253-1 standard. 


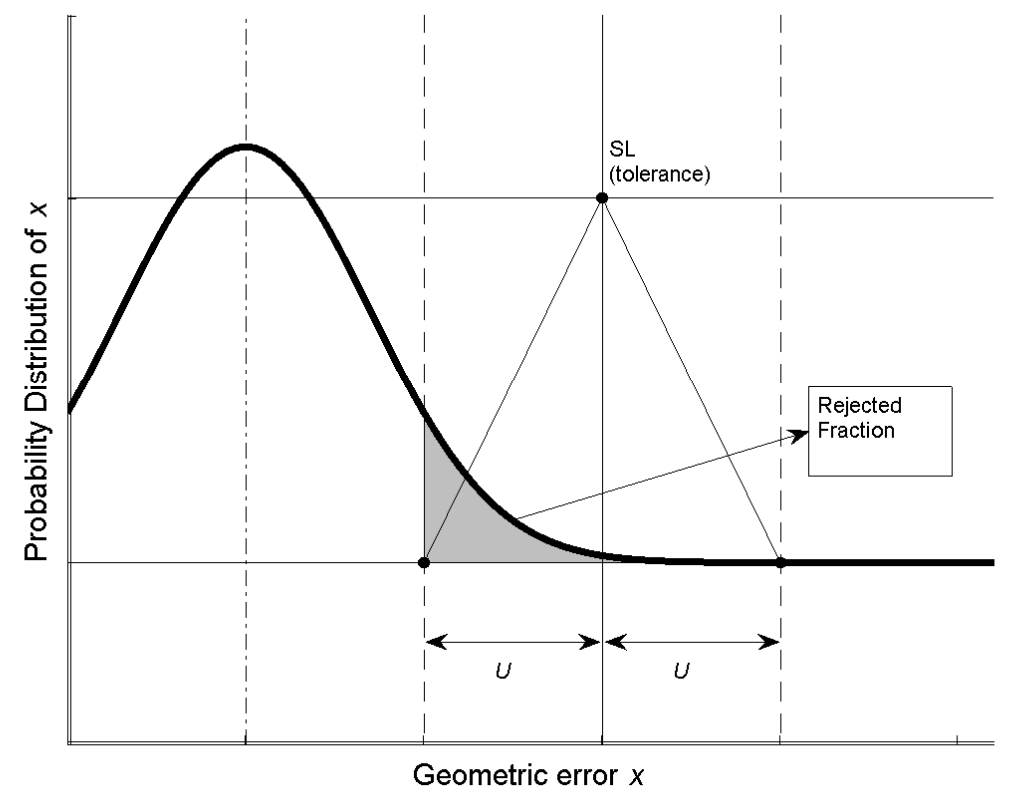

Figure 2: Wrongly rejected fraction when the conformance test is applied. 


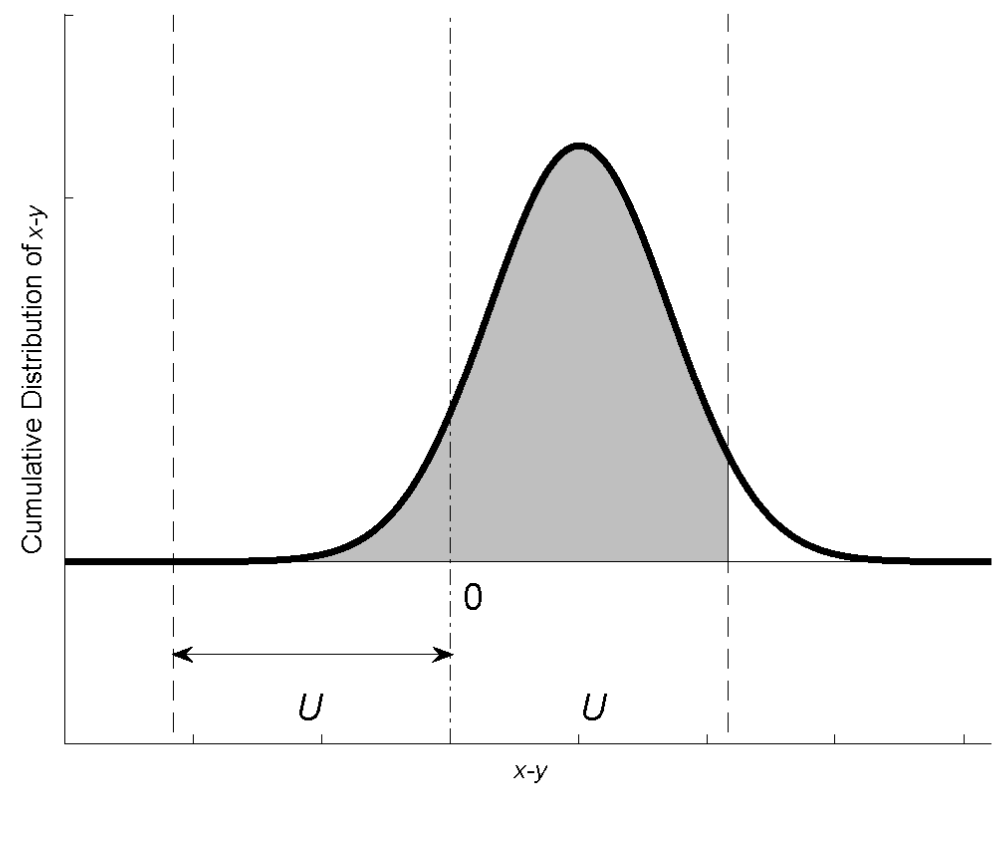

Figure 3: Simulation based uncertainty evaluation. 


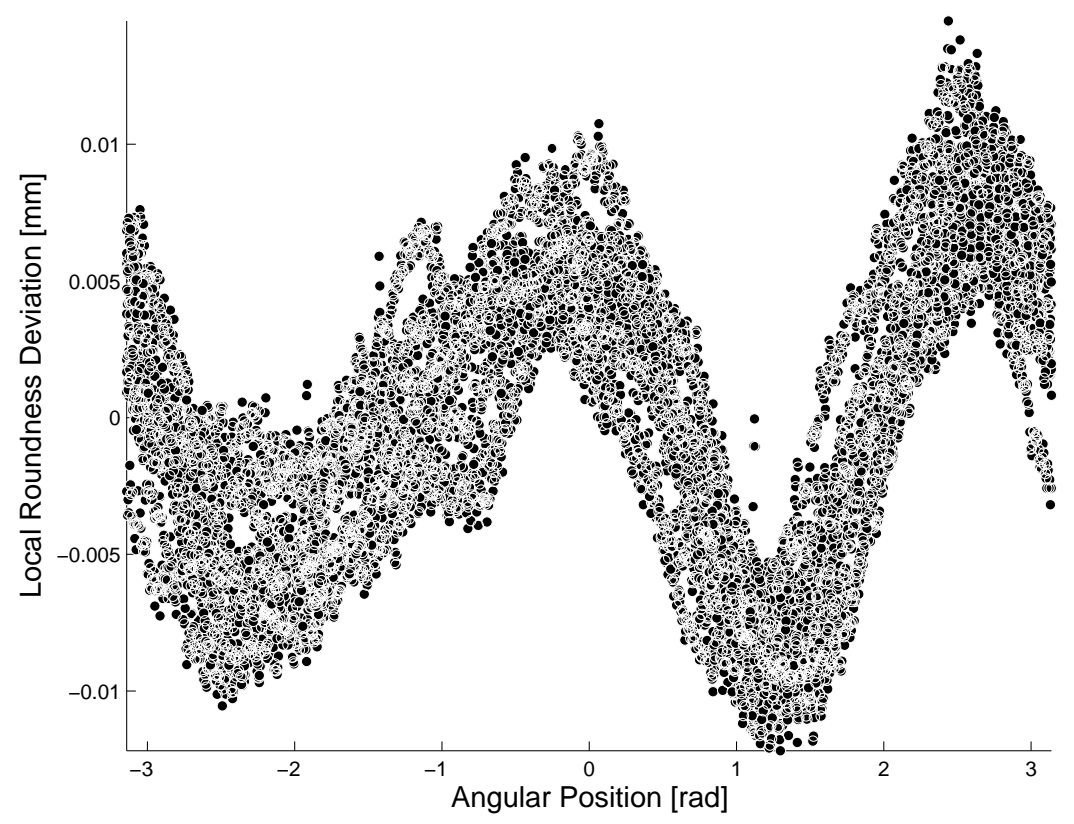

Figure 4: Manufacturing signature of titanium alloy specimen - plot of the residual from an ideal circumference of the ten titanium alloy specimens roundness profiles. 


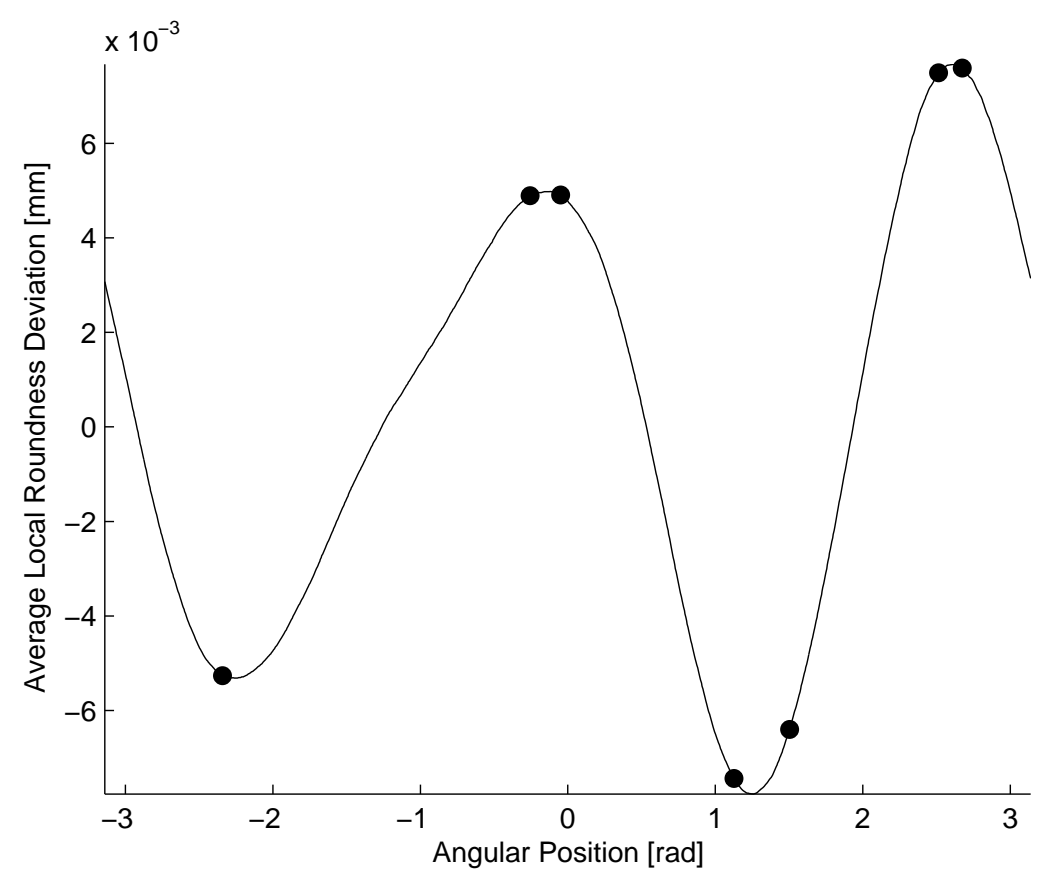

Figure 5: Titanium roundness manufacturing based sampling strategy. 


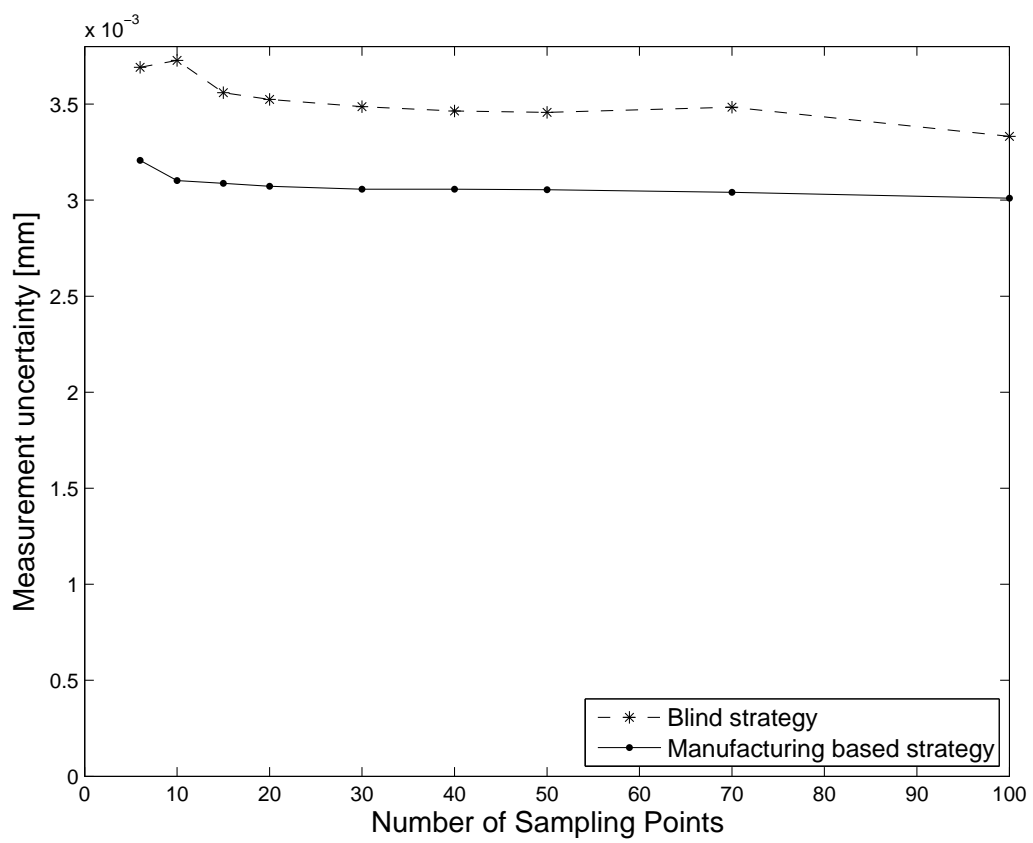

Figure 6: Measurement uncertainty in titanium specimens roundness measurement. 


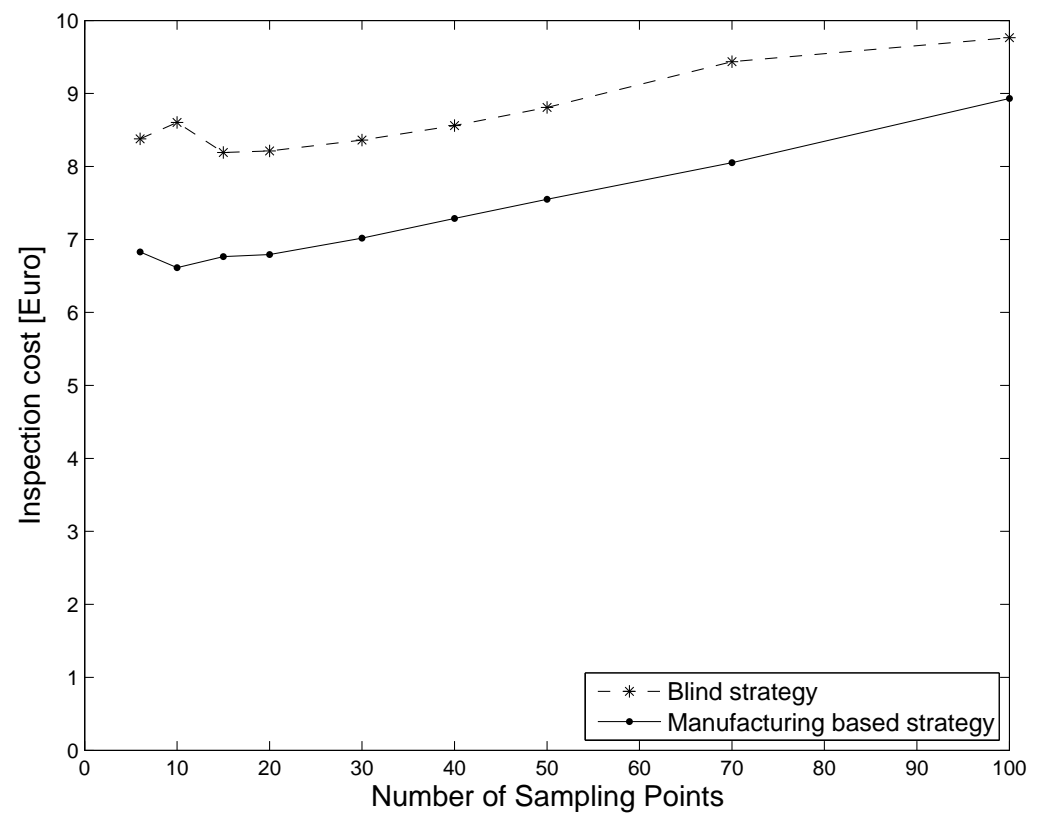

Figure 7: Inspection cost in titanium specimens roundness measurement. 


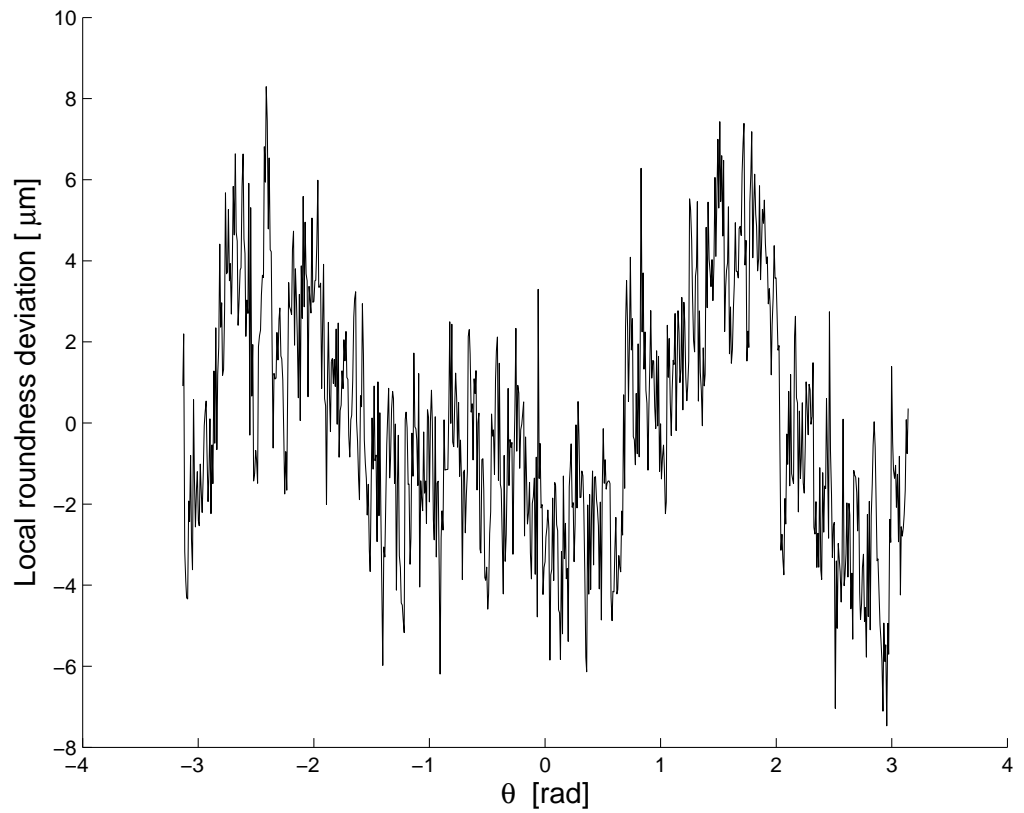

Figure 8: Exemple of simulated roundness profile. 


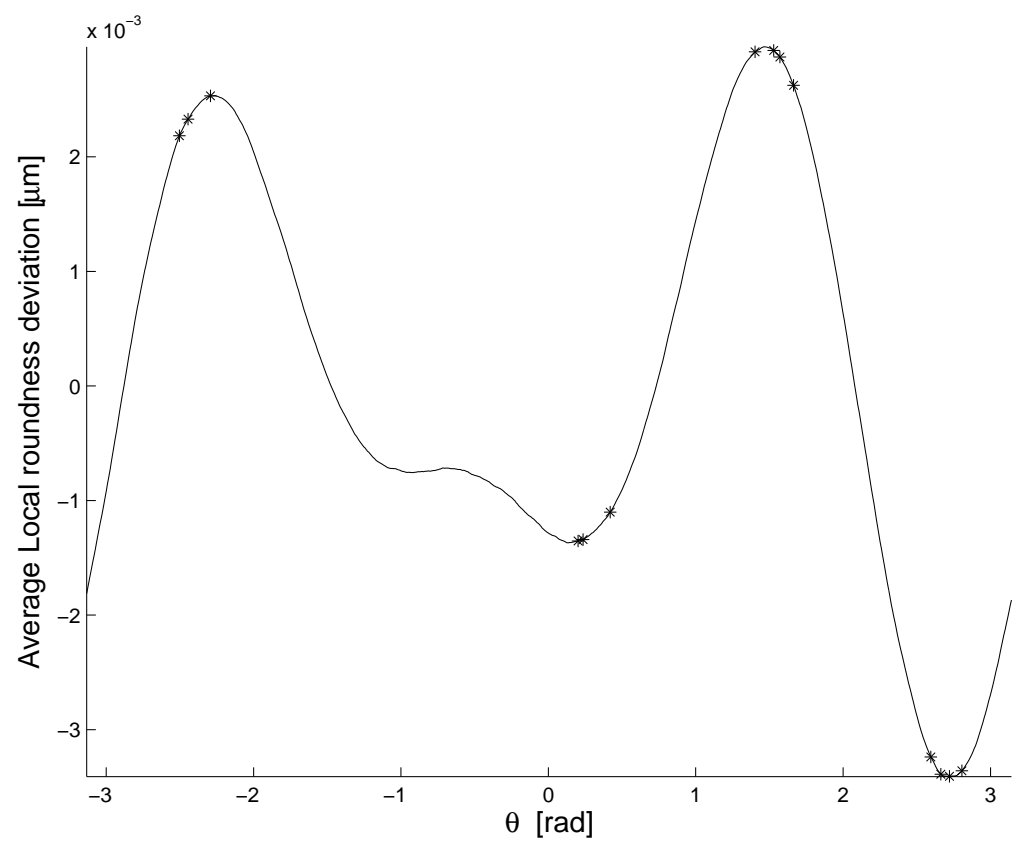

Figure 9: Average profile and optimal roundness strategy (simulation approach). 


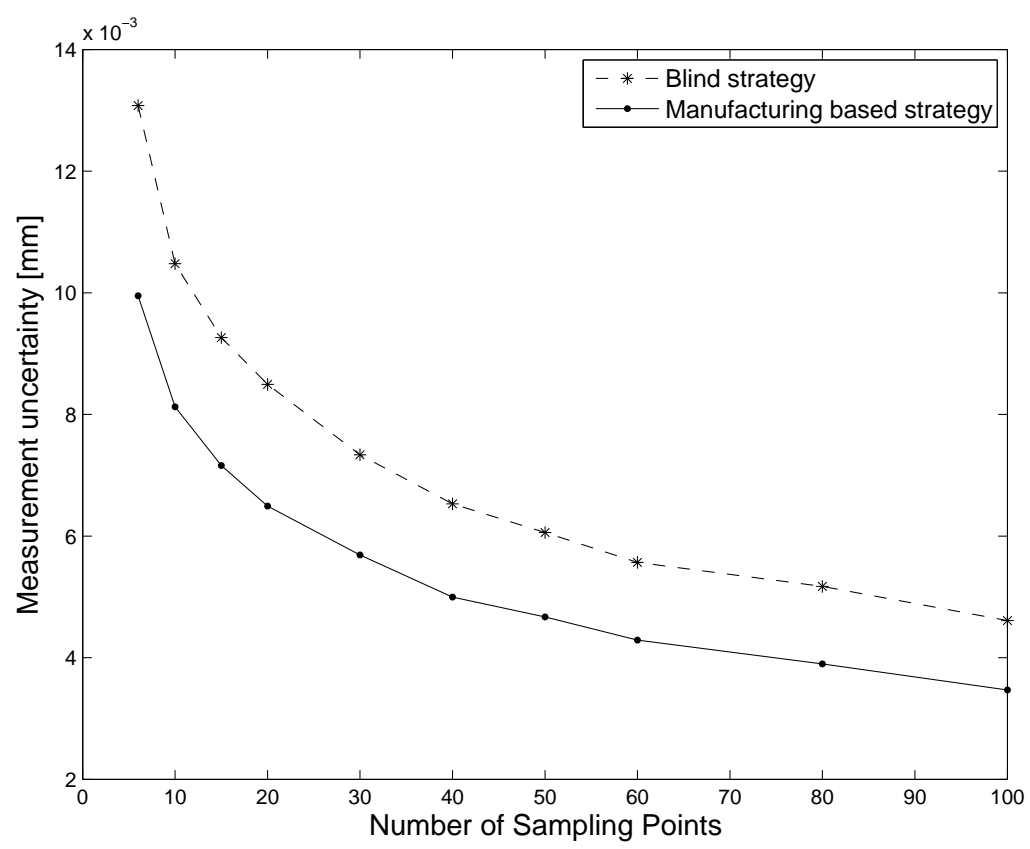

Figure 10: Measurement uncertainty in steel specimens roundness measurement. 


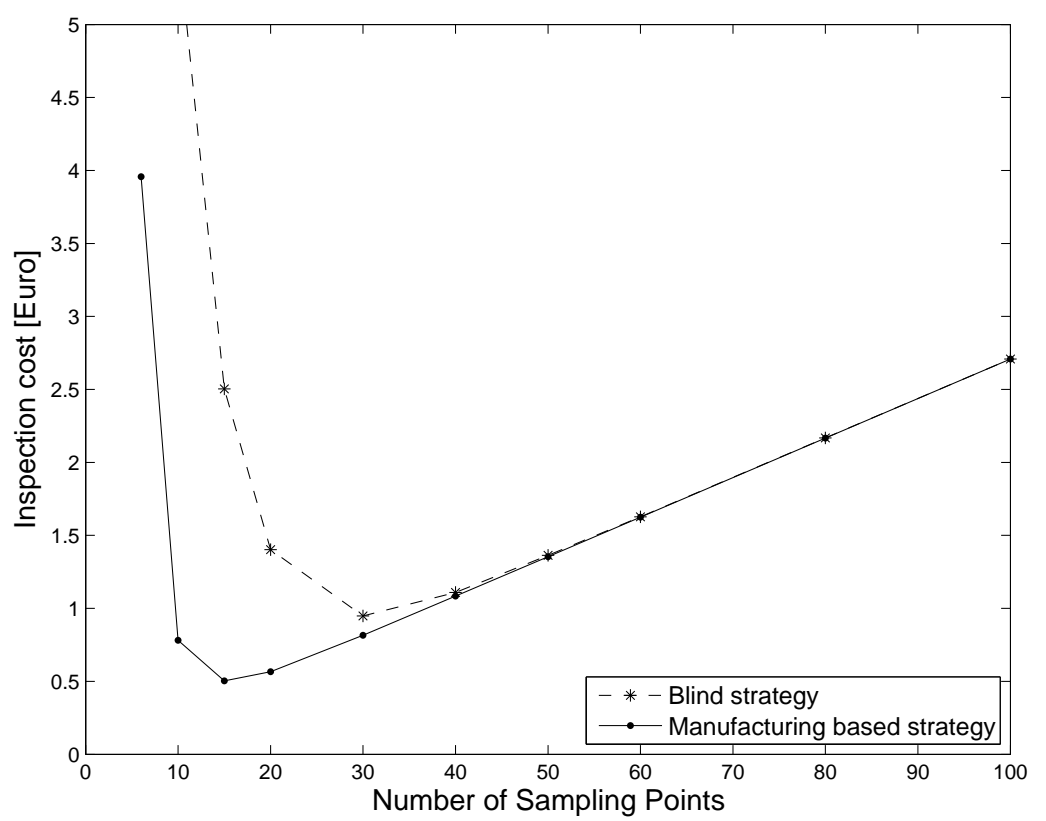

Figure 11: Inspection cost in steel specimens roundness measurement. 
a)

Figure 12: Average surface of ten toleranced planes. 


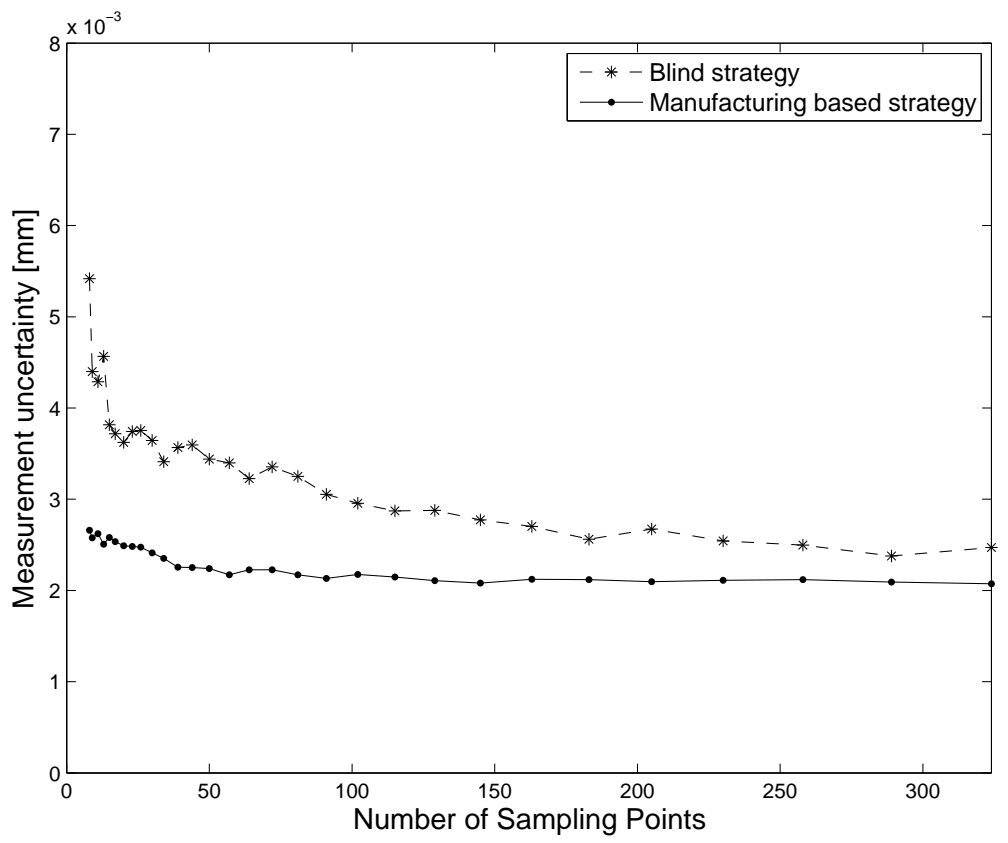

Figure 13: Measurement expanded uncertainty as the sample size increases for the parallelism of the aluminum specimens (artifact based approach). 


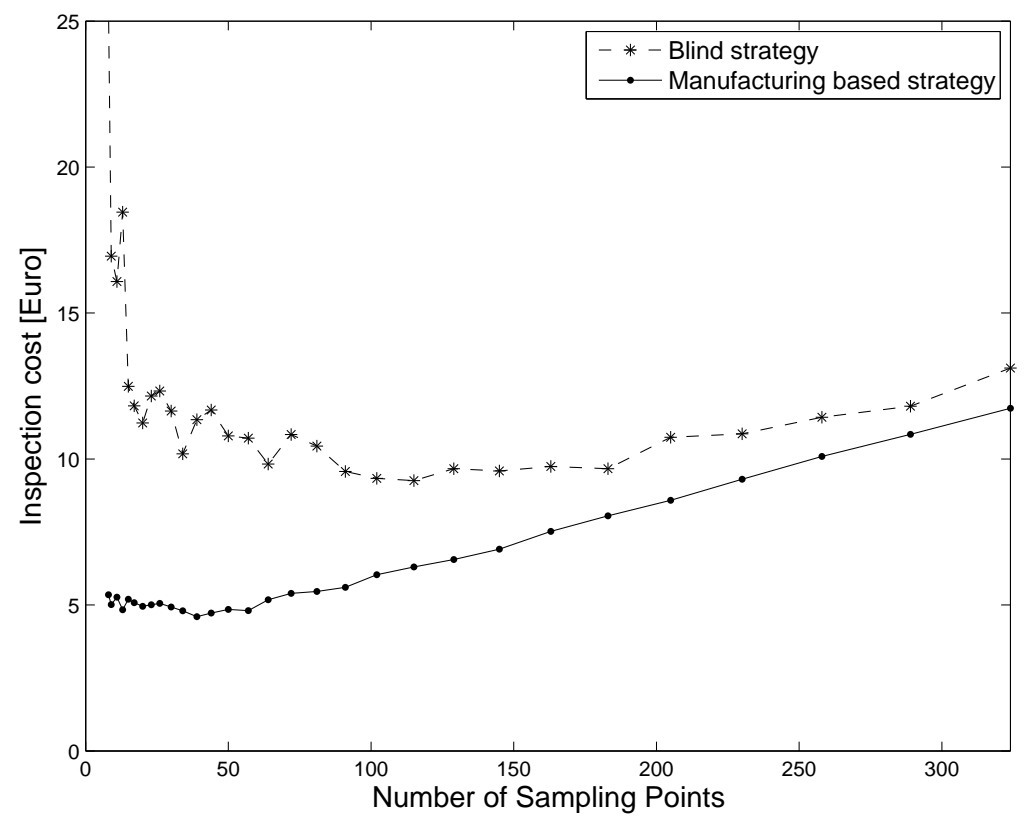

Figure 14: Inspection cost as the sample size increases for the parallelism of the aluminum specimens (artifact based approach). 


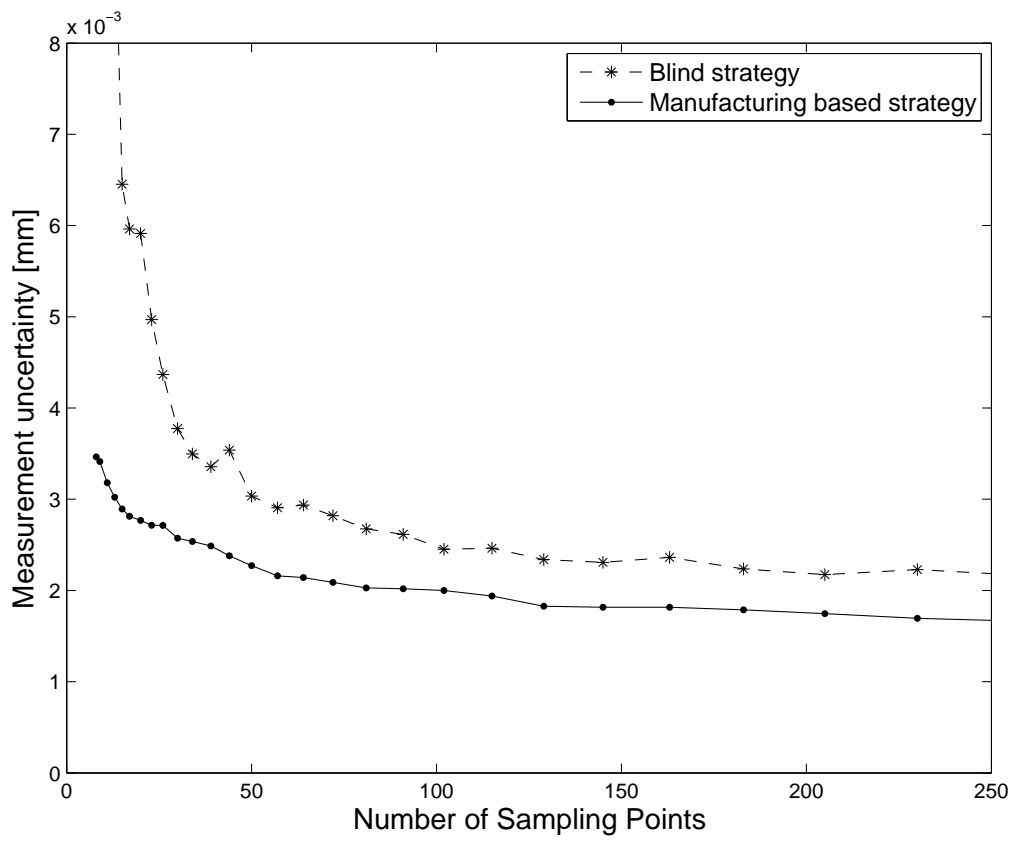

Figure 15: Measurement expanded uncertainty as the sample size increases for the parallelism of the aluminum specimens (simulation based approach). 


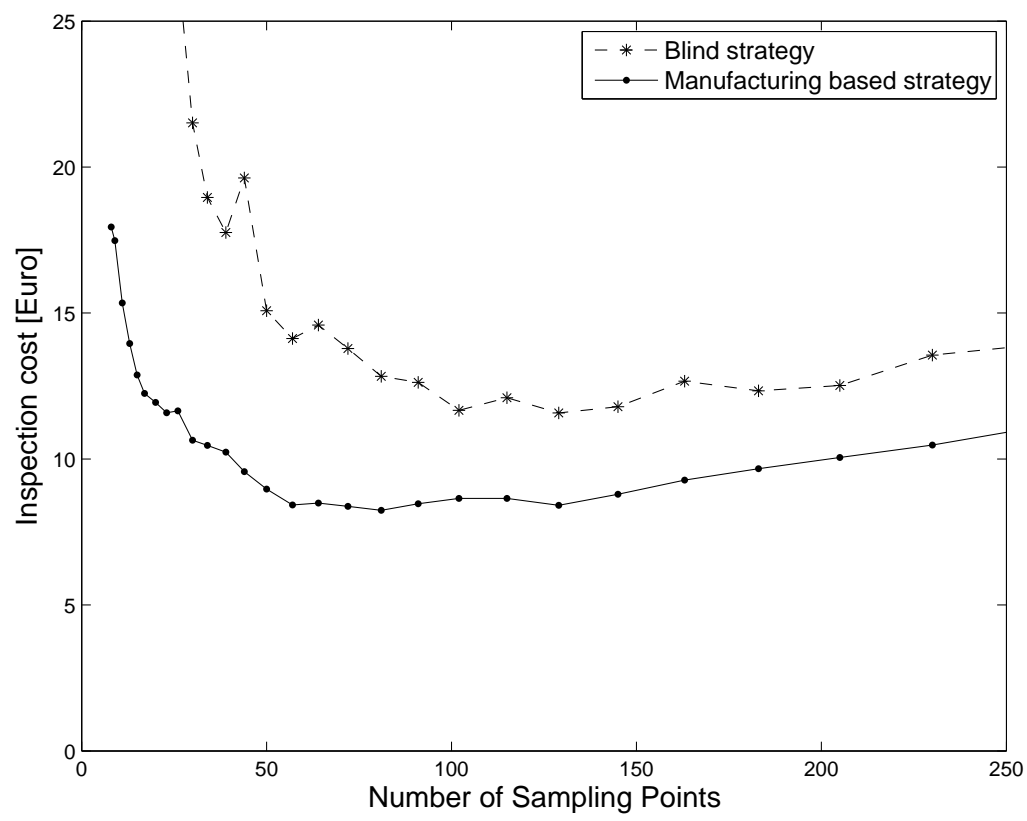

Figure 16: Inspection cost as the sample size increases for the parallelism of the aluminum specimens (simulation based approach). 